\title{
A Level-Wise Periodic Tree Construction Mechanism for Sleep Scheduling in WSN
}

\author{
Nachiketa Tarasia, Amulya Ratna Swain, Soham Roy, and Udit Narayana Kar
}

\begin{abstract}
The wireless sensor network (WSN) has been extensively used to monitor and control the natural ecosystem on a large scale like air quality, natural life, etc. Low battery power, small storage, and limited processing ability are the most critical areas of concern in WSN. To reduce energy utilization, the sensor nodes in WSN work in a cyclic process between active and sleep mode. A certain number of nodes are chosen active, and they are responsible for sensing as well as data transmission, and the rest of the nodes are gone to sleep. With the objective to lengthen the durability of the network, we have presented a level wise periodic tree construction algorithm. The algorithm uses a specific set of nodes to participate in tree construction. To minimize the overall energy consumption, all nodes do not need to participate in transmission and reception. As per the suggested procedure, the main goal is to put the nodes, which are currently active and have already spent a significant amount of energy, to sleep mode. The proposed approach will provide a chance to the leaf nodes to become active and uphold the connectivity. The performance of the proposed protocol is evaluated using the Castalia simulator. The simulation results show that the proposed level wise periodic tree construction approach increases the durability of the network in conjunction with the non level approach.
\end{abstract}

Index Terms-WSN, Sleep scheduling, Energy Efficiency, Hierarchical Structure.

\section{INTRODUCTION}

In recent decades, continuous upbringing in terms of sensing, storage, and communication have enabled wireless sensor networks (WSNs) to be used in a diversified manner. Minute observance of atmospheric conditions, monitoring the natural life, biomedical surveillance, calamity detection, etc., are some of the major applications of WSNs. Deployment of a larger number of sensor nodes within a network leads to the execution of bottleneck. The primary concerns behind the bottleneck execution are minimum battery power, minimal memory and lower computational capacity of sensor nodes [1], [2]. Therefore, reduction in continuous data transmission can enhance the overall network lifetime [3], [4]. In such type of scenarios, clustering algorithms can play a major role [5], [6].

To have the maximum coverage and network dependability, most of the WSN applications need an enormous number of nodes to be deployed densely. The dense deployment of the sensor nodes expands the quality of transmission. At the same

Manuscript received December 15, 2019; revised April 5, 2020. Date of publication June 8, 2020. Date of current version June 8, 2020.

Authors are with the School of Computer Engineering, Kalinga Institure of Industrial Technology, Bhubaneswar, Odisha, 751003, India (e-mails: \{ntarasia06, swainamulya, skr2538, uditnarayankar\}@gmail.com).

Digital Object Identifier (DOI): 10.24138/jcomss.v16i2.974 time, the network would not get exhausted in terms of energy. The minimal power in densely deployed sensor nodes affects the overall network lifetime. To minimize the overall energy consumption of a network, WSNs operate in a mandatory cycle mode. In mandatory cycle mode, the sensor nodes can effectively toggle in between active and sleep states [7], [29]. The nodes, when going to the sleep state, switch off their own radios to conserve power. Similarly, the nodes in the active state switch on their radio. This mechanism enables a lesser number of nodes to stay active throughout the entire network. For each estimation cycle, more than a few active sensor nodes are chosen. Hence, the overall procedure is quite energy efficient and productive.

The selection of active nodes in WSN must satisfy two basic requirements, i.e., coverage and connectivity. While covering an area of interest, the deactivation of a few sensor nodes may save energy. Nevertheless, it should ensure that no regions should leave uncovered. Appropriate management in covering an area of interest is, however, vital as far as overall power consumption is concerned. This can enhance the lifetime of the network. On the other side, the data sensed by the active nodes must be reached at the sink node for further use, which requires connectivity among the active nodes. The mode of communication among the active nodes can either be single hop or multi hop. The overall transmission cost is directly proportional to the number of active sensor nodes. The transmission cost goes higher if the number of active nodes within a network increases. Therefore, it is required to make a few nodes active for data transmission and ensuring connectivity.

In this manuscript, we have proposed a sleep scheduling mechanism that reduces the overall energy consumption in a WSN with ensuring network connectivity. The proposed approach makes use of the hierarchical structure, which is constructed periodically in parts rather than for the whole network with a target to minimize energy consumption. The rest of the paper is organized as follows. Section II presents the state of art. Section III presents the proposed approach to enhance network longevity. The detailed simulation and generated results are presented in Section IV. The manuscript concludes in Section V.

\section{STATE OF ART}

Nowadays, an infinite number of applications are dependent upon WSNs. Basically, in a densely deployed WSN, the energy consumption of the sensor nodes is high. This has a direct 
impact on the overall network lifetime. Looking at this issue, researchers have invested a great deal of time in finding the solution. Hence, there is an abundance of research papers available in the background. In this section, we present an in depth literature study related to a reduction in energy consumption in WSNs.

The construction of hierarchical structure plays a vital role in designing different media access control (MAC) and routing protocols for WSN [36], [28]. Considering this fact, Dash et al.[29] proposed a distributed algorithm[] to construct the hierarchical structure where each node maintains two parents expect the sink node. The selection of parent nodes is mostly based on their remaining energy. Here, the authors claim that the energy consumption over the whole network has considerably reduced as the approach makes more nodes to be in and sleep and also due to its distributed nature.

A combination of connectivity and complete in WSN is proposed in [8], [28]. The proposed approach seemed to be quite useful in enhancing the overall network lifetime. It supported $k$ level coverage and connectivity in the desired area. Based on the problem requirement, the level of monitoring through sensor nodes was modified. To check whether the convergence point of the parameter of the detecting region is being covered by the excess number of sensor nodes or not, a strategy for minimizing the total number of active nodes is being proposed. The sensor node is said to be an additional sensor node if and only if all the convergence points of the parameter are covered by neighboring nodes. Initially, all the sensor nodes must be in $O F F$ state. Each sensor node inside a network carries a qualification calculation. The qualification calculation signifies the event based on which the node should go to $O N$ state or remain in $O F F$ state. As per the proposed approach, the transmission range of the sensor nodes becomes multiple times higher than that of the detection range. At the same point of time, the overall coverage verification of the network is also adequate. The network lifetime is based on the energy of each and every sensor node. Even if a single node fails, the network can be split. To enhance the stainability of the node and increase the lifetime of the network, the proper transmission path must be chosen. Potentiality timeserving reliable routing (PTTR) method utilizes the acknowledged idea and guarantees the conveyance of packets as well [13]. The packet traverses from one hop to another hop until it reaches the destination. The next hop node is decided by factors like remaining energy, link duration, and the distance it needs to travel. The source node transmits the aggregate data to the best next hop node. Promptly, the best next hop node sends the acknowledgment back to the sender. Likewise, an equation for Steadfast Routing Factor (SRF) is additionally proposed in SRIR [14], which utilizes energy, distance, and PRR to help in choosing a node as the next hop node. The path is set up by each sensor nodes dynamically towards the sink node similarly in a hop to hop way. In EARQ [15], a node calculates the remaining energy costs, postponement, and performance of the sink node route, construct just with respect to data from neighboring nodes. The likelihood of choosing a path by the node is measured. Whenever there is a requirement of sending a packet is required, the next hop node is randomly selected. A path with lower remaining energy costs is likely to be chosen because the probability is contrary to the remaining energy costs of the sink node. To ensure the optimized density of the sleep nodes and to maintain the overall coverage at the same time, a decentralized algorithm is proposed in [9]. The proposed approach focused on covering the desired area, setting up the connectivity, and enhancing the network lifetime. The region in which the sensor nodes are placed is carved into a grid structure. If the grid center is covered up by the neighboring sensor nodes, the sensor node in the center can go to sleep. To check if the grid center is covered, the sensor node receives an $O N$ message. If the center of the grid is covered, the sensor node can go to the sleep state. In specific applications, for e.g., temperature monitoring continuous screening of the coverage area is not necessary. Such applications need partial coverage for an area of interest. A demand driven connectivity and coverage mechanism for WSNs is suggested in [10]. The sensing range for the sensor nodes is adjusted, looking after the required coverage. The authors proposed a local route optimization algorithm to minimize the overall power consumption in the network. Better topology control algorithms integrated with enhanced sleep scheduling mechanisms can enhance the overall lifetime in WSNs [26]. Considering the essential factors like coverage, connectivity, and communication $(C 3)$, a holistic approach has been proposed in [11]. The proposed algorithm worked in 4 stages as enlisted. 1) Isolated the network into virtual rings by using received signal strength indicator (RSSI) 2) characterized the rings with group heads at substituting rings 3) made rings inside the bunches utilizing the triangular decoration and 4) to send the information through group heads. The obtained results ensured partial coverage, one connectivity, and energy efficient communication and 90\% coverage as per the availability of the sensor nodes.

The framework suggested in [12] clarified the sleep and wake up procedures of the fuzzy clustering algorithm. Initially, all the sensor nodes in the network remained in the sleep state. Once the data transmission begins, the source node communicates the packet to the forwarder array. The packet acts as an alert message and triggers other nodes present inside the network. This generates a list of nodes with maximum battery power. The nodes with maximum energy stay active while other nodes go to sleep state. Hence, the overall energy consumption of the network is minimized. Energy efficient algorithms for the selection of nodes have been introduced for WSNs [16]. A new Correlated Node Set (CNS) and a High Residual Energy First (HREF) node determination calculation decrease the active node number. The Cover Sets Balance calculation (CSB) is fit for picking active nodes; those with high coverage range and high outstanding remaining energy. In every active node determination step, the coverage range and remaining energy are utilized to locate an underlying active node set and then adjust the span of the cover sets, keeping in mind that the end goal is to remove low remaining energy nodes.

WSN's path selection strategy is regularly guided by two basic prerequisites: limiting energy costs or increasing network output. Conventional wired system path selection protocols 
select the best hubs location between source and destination and forward each packet through that cluster. This approach has generally been followed by most path selection protocols designed for multihop WSNs, and such multi path routing protocols are included. In any event, this did not take into account points of interest for the broadcast nature of wireless communications, i.e., the transmission of a node could be heard by any node within its transmission area. In WSNs, different elements, such as blurring, impedance, and multi path impacts, can prompt transitory overwhelming packet losses in a preselected path [17]. Many energy efficient path selection protocols [18], [19] have been proposed as of late joining with an assortment technique. Most of the latest power conscious methodology did not consider the wireless connections packet failure, i.e., packet loss. An attempt has been made to reduce energy usage by distributing energy absorption among cluster participants. Topology control studies [20], [21], [22] focus on network connectivity and blockiness while improving different design goals. Paper [23] presented a path selection protocol called energy efficient opportunistic routing algorithm (EEOR). The proposed protocol chooses the forwarder set from the neighboring set of nodes and prioritizes them in the light of the energy saving arrangement. In order to address this issue, a large number of protocols have been revised. From the above discussion, it is clear that in a WSN, almost any single node is involved, whether the packet is transmitted or in an idle state. Despite there is no involvement of active nodes in any transmission, they still get exhausted in terms of battery power [30]. The sleep mode, in particular, is actualized to conserve energy. The sleep mode additionally decreases energy utilization. As per the mechanism proposed in [24], it can be seen that sleep mode idea can be actualized in various courses with the assistance of queue network, RTS, and CTS signals or wakeup caution radio idea.

A scheduling based energy aware routing protocol has been presented in [25]. In the proposed approach, the sensor nodes are deployed in a tree structure. As per the mechanism, the sensor nodes are divided into two groups, i.e., internal nodes and leaf nodes. For successful transmission of data, the internal nodes remain active, whereas the leaf nodes stay deactivated. However, the internal nodes are the sensor nodes with maximum battery capacity, and leaf nodes have lower battery capacity. The leaf nodes become active only when they sense some activity in their surrounding. After collecting the data from a certain activity and transmitting the same, the leaf node again returns to sleep mode. In the tree structure, every node has a dedicated parent node, and whenever it senses or receives any data, it always forwards the same to its parent node, except the sink node. This is an iterative process, and it continues till sensed data is transmitted to the sink node. However, after this phase of tree construction and data transmission, there is a significant energy gap between the active internal nodes and the leaf nodes. As a result, the internal nodes may die out quickly as compared to the leaf nodes that result in maintaining connectivity of the tree is short lived. Therefore, it is important to equally maintain energy dissipation in all the nodes of the WSN so that the connectivity can last longer.
The duty cycle is one of the most widely used energy saving mechanisms for WSNs. To maximize the overall network lifetime, the duty cycle mechanism ensembles sleep and wake up time of sensor nodes. An adaptive duty cycle mechanism for MAC protocol in WSNs is proposed in [32]. These adaptive scheme judges the listen time of each node involved in packet transmission and reception scenarios. The proposed approach reduced the waiting time for each transmitted packets and undelivered packets as well. Hence, the overall energy consumption is reduced.

As per the existing literature, the primary focus is on the non sleeping scenarios. The underlying assumption made in most of the pieces of literature says that the nodes in a WSN communicate using a single channel. This mechanism, when embedded in a multi channel asynchronous duty cycle scenario, reduces the performance of the WSNs. To address this issue, a delay efficient data aggregation scheduling problem for multi channel asynchronous duty cycled WSNs is proposed in [33]. The proposed approach is based on two concepts, namely, candidate active conflict graphs (CACGs) and feasible active conflict graphs (FACGs). Using these concepts, the authors proposed an efficient data aggregation scheduling (EDAS)algorithm for WSNs. The proposed algorithm uses the fewest child first rule to choose the forwarding nodes. The overall efficiency of the algorithm is proved through extensive simulation and analysis.

The nodes involved in the transmission of data packets sometimes transmit the redundant data. This is a complete wastage of time and energy. To resolve this issue, an energy efficient sleep scheduling mechanism is presented in [34]. For a reduction in energy consumption, the nodes in WSN is scheduled as active or sleep mode. To organize all the sensor nodes into a cluster, the optimal competition radius is computed. Based on the collected data by the member nodes, A fuzzy matrix is generated. The obtained fuzzy matrix measures the similarity degree of the member nodes. Using the correlation function and the similarity degree, the sensor nodes are divided into different categories. To maintain data integrity, the redundant nodes are selected and put into a sleep state.

In a large scale, WSNs, where the nodes are clustered in the form of a tree structure, may suffer from higher end to end delay. At the same time, the congestion within the network remains high as well. To resolve this issue, and to maximize the converge cast traffic in large scale WSNs, an efficient mechanism is proposed in [35]. The proposed mechanism is the combination of the data generation mechanism of the individual nodes and efficient allocation schemes of the active periods of the sensor nodes.

\section{PROpOSED APPROACH}

As discussed earlier, the internal sensor nodes deployed in a tree structure remains active. After spending a significant amount of energy, the internal nodes go to sleep mode. This allows the leaf nodes to maintain network connectivity. To perform this mechanism, periodic tree reconstruction is needed. In every reconstruction phase, the intermediate nodes 
TABLE I

NOTATIONS

\begin{tabular}{|c|c|}
\hline Short Name & Description \\
\hline$n_{i}$ & $i^{t h}$ node \\
\hline$S S(i)$ & Sleep signal for $i^{t h}$ node \\
\hline$R R(i)$ & Remaining energy of $i^{t h}$ node \\
\hline$L E V E L(i)$ & Level of $i^{t h}$ node \\
\hline$P T(i)$ & $\begin{array}{l}\text { No. of packets transfer by } i^{t h} \text { node in the } \\
\text { current round }\end{array}$ \\
\hline$A L I V E(i)$ & True if $i^{t h}$ node is alive \\
\hline$C O N S(i)$ & No. of consecutive rounds node $i$ is active \\
\hline$C R L(n)$ & $\begin{array}{l}\text { Current level reconstruct for the } n^{t h} \text { round. } \\
C R L(n) \quad(C R L(n-1)+ \\
1) \% \text { max level. } \\
\text { A CRL value of } 3 \text { means level 1,2,3 will be } \\
\text { reconstructed. }\end{array}$ \\
\hline$P F_{j, 1}$ & 1st parent field of node $\mathrm{j}$ \\
\hline$P F_{j, 2}$ & 2nd parent field of node $\mathrm{j}$ \\
\hline
\end{tabular}

and the leaf nodes are interchanged. It signifies that, in every two consecutive reconstruction phases, the leaf nodes with higher energy becomes the internal node. Once the internal nodes get exhausted in terms of power, it becomes the leaf node in the next phase. In this mechanism, the overall energy consumption of the nodes is reduced. However, it can not be ruled out that the nodes which are closer to the sink node will expend more energy and thus exhaust sooner than the nodes far away from the sink node.

To overcome this problem and enhance the network lifetime, we hereby propose a level wise tree construction mechanism. As per our algorithm, each and every node does not need to get involved in the tree reconstruction phase. Hence, the nodes which are at a larger distance from the sink nodes get less exhausted in comparison to the nodes nearer to the sink. Therefore, in each reconstruction phase, it is not necessary for all the nodes to participate. The upper level nodes will participate more during the reconstruction phase as compared to the lower level nodes. So, in any reconstruction phase, if level-1 nodes will participate in reconstruction, then in the next round level-1 and level-2 will participate in reconstructing and in the next round level-1, level-2, level-3 and so on. Once the nodes belong to all the levels participate in the reconstruction phase, then the nodes can again begin where only level- 1 nodes will participate. In the tree reconstruction phase, the nodes which are active in the $n^{t h}$ round has a high probability of going to sleep state in $(n+1)^{t h}$ round. Nevertheless, the time duration between two consecutive reconstruction phase for nodes which are away from the sink is more than that for nodes which are nearer to the sink. This proposed approach uses a systematic structure of message passing among the nodes to mend the tree locally and thus using a minimum amount of energy to reconstruct without involving all the nodes of the WSN. Any active parent node which wants to go to sleep in the next round needs to find an alternate parent for its children, and else it will not be allowed to go to sleep mode. There exist 4 cases as given below. The symbols used for different paths are shown in figure 1 . The notations used in the proposed protocol are given in table I.

1) Both $P(n+1)$ and $P(n)$ received new parent, i.e. node in $(n+1)^{t h}$ layer and node in $n^{t h}$ layer received new parent. In figure 2, initially, the parent of $P(n+1)$ is $P(n)$ and that of $P(n)$ is $P(n-1) . P(n+1)$ found another node as new parent and $P(n)$ found another node as its new parent. So, $P(n-1)$ and $P(n)$ can go to sleep.

2) $P(n)$ is able to find new parent but $P(n+1)$ is unable to find. In figure 3, $P(n+1)$ is unable to find new parent where as $P(n)$ is able to find a node as its new parent. So, $P(n+1)$ can go to sleep.

3) Both $P(n+1)$ and $P(n)$ are unable to find new parent. In figure 4, both $P(n+1)$ and $P(n)$ cannot go to sleep. They have to stay active.

4) $P(n+1)$ is able to find new parent but $P(n)$ is unable to find new parent. In figure $5, P(n+1)$ is able to find an new parent. But $P(n)$ is unable to find new parent. So, $P(n)$ can go to sleep, but $P(n-1)$ cannot.

Initially, all the nodes are in active mode. The re-construction of the tree happens after the completion of each round. So, after the $n^{t h}$ round is finished, the reconstruction phase of $(n+1)^{t h}$ round begins.

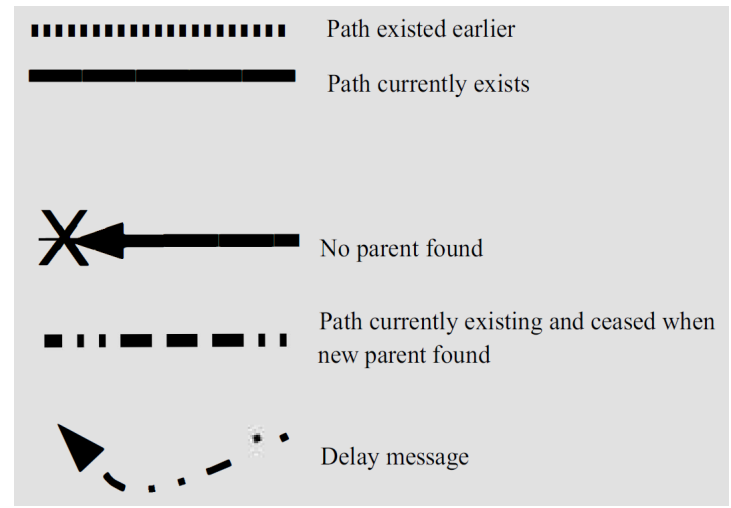

Fig. 1. Symbols used for different path

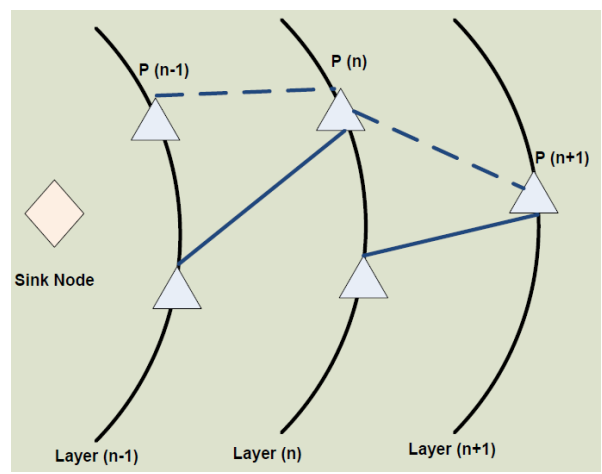

Fig. 2. Case 1:- Both $\mathrm{P}(\mathrm{n}+1)$ and $\mathrm{P}(\mathrm{n})$ received new parent. So, $\mathrm{P} 2$ and $\mathrm{P} 1$ can both go to sleep.

At the very beginning, $C O N S(i)$ and $P T(i)$ of each node is set to zero. An active node $n_{i}$ can make a request to go to sleep for the $(n+1)^{t h}$ round, if in the $n^{t h}$ round $P T(i)$ is greater than the threshold value or $C O N S(i)$ is equal to a fixed value. Here, we set the fixed value to be 3 . Also, the active node $n_{i}$ must be within the CRL(n) to initiate its 


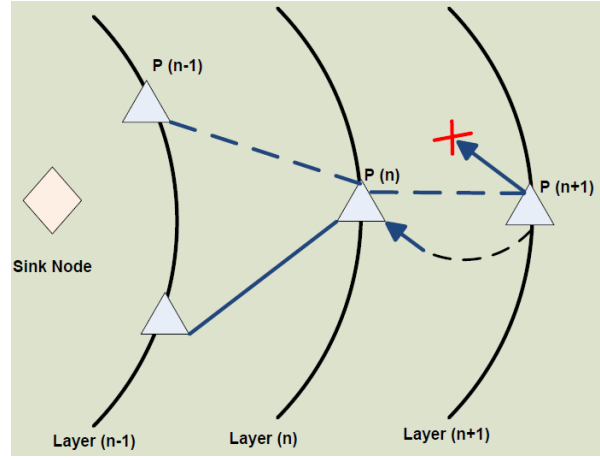

Fig. 3. Case 2:- $P(n)$ is able to find new parent but $P(n+1)$ can not.

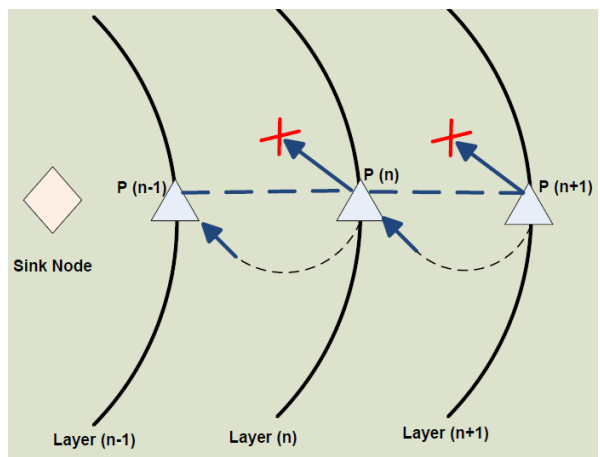

Fig. 4. Case 3:- Both $\mathrm{P}(\mathrm{n}+1)$ and $\mathrm{P}(\mathrm{n})$ unable to find new parent.

sleeping request process. The sink node always remains active during the entire lifetime of WSN.

After an active node, $n_{i}$ triggers a process to go to sleep, $S S(i)$ is turned on. The tree reconstruction starts with broadcasting the FIND_PARENT message packet to its children. The FIND_PARENT message packet instructs the child nodes of $n_{i}$ to find a new parent. The active node $n_{i}$ waits for a random amount of time until all its children get a new parent before it goes to sleep. Once a node $n_{j}$, which is a child of $n_{i}$ receives the FIND_PARENT packet, it initiates its process to find a new parent by broadcasting a WANT_PARENT packet and waits for a random time while it chooses the best parent node from the reply of the other nodes.

A node $n_{k}$, which receives a WANT_PARENT packet, is eligible to be a parent only when $S S(k)$ is false and $A L I V E(k)$ is true. Once it meets its eligibility criteria it sends out a PARENT_REPLY packet with information such as $L E V E L(k)$ and $R R(k)$. The node $n_{k}$ is ready to become a parent of any node if it is chosen by a node $n_{j}$, which is looking for a parent. A node $n_{j}$, which is looking to find a new parent might receive multiple PARENT_REPLY packets. Out of all the PARENT_REPLY packets from its neighboring nodes, it chooses the most suitable node, $n_{k}$, to be its parent based on the following conditions. A node $n_{j}$ will give a higher priority to a node $n_{k}$ to be its parent when $L E V E L(k)$ is equal to $L E V E L(j)-1$ rather than $L E V E L(k)$ is equal to $L E V E L(j)$. Nevertheless, node $n_{j}$ will out rightly reject all nodes $n_{k}$ when $L E V E L(k)$ is less than $L E V E L(j)$.

After propagating through this initial choosing criteria, $n_{j}$ employs another criterion where it selects its parent based

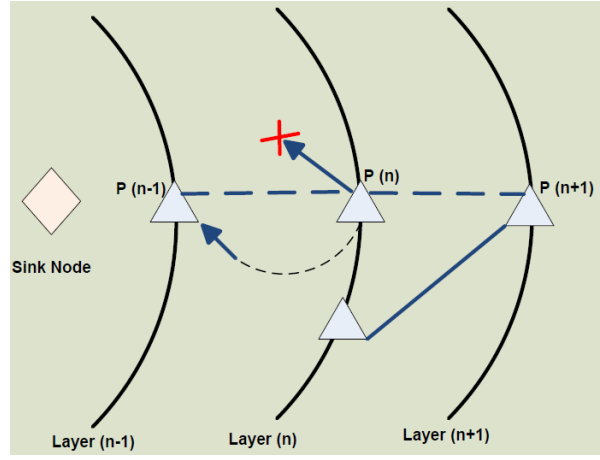

Fig. 5. Case 4:- $p(n+1)$ found new parent but $P(n)$ can not.

on the remaining energy of the nodes. It chooses $n(k 1)$ over $n(k 2)$ if $R R(k 1)$ is greater than $R R(k 2)$ provided $L E V E L(k 1)$ is equal to $L E V E L(k 2)$. After node $n_{j}$ selects its parent from the multiple PARENT_REPLY packets, it needs to notify its new parent $n_{k}$ of its new selection. So, it broadcasts an ACKNOWLEDGEMENT packet to $n_{k}$. When $n_{k}$ receives the ACKNOWLEDGEMENT packet, it becomes an internal node and turns active for the $(n+1)^{t h}$ round as it is some other nodes parent now.

Once this process gets over, node $n_{i}$ waits for a random time to check whether all of its children have received their new parent. So, it broadcasts a FIND_CHILD packet into the network. When a node $n_{j}$ which remains the child of $n_{i}$ receives the FIND_CHILD packet from $n_{i}$, it broadcasts a CHILD_REPLY message to $n_{i}$ to notify that $n_{i}$ cannot go to sleep for the $(n+1)^{t h}$ round as it did not get rid of all its child nodes. When $n_{i}$ receives the CHILD_REPLY message, it sends a special MINUS_ONE message to its child $n_{j}$ instructing the child $n_{j}$ to use the alternative $(P F)_{j, 2}$ as its primary parent and set the $(P F)_{j, 1}$ to -1 . In this way, $n_{i}$ tries to get rid of its child $n_{j}$.

If this process fails to eliminate $n_{j}$ as child of $n_{i}$, it has to remain active for the $(n+1)^{t h}$ round. $n_{i}$ again tries to go to sleep for the $(n+2)^{t h}$ round after the completion of the $(n+1)^{t h}$ round. Nevertheless, if every child $n_{j}$ of $n_{i}$ is able to receive a new parent, i.e. $n_{i}$ does not receive any CHILD_REPLY packet, then in that scenario $n_{i}$ can go to sleep for the $(n+1)^{t h}$ round and CONS(i) is reset to 0 . When the $(n+1)^{t h}$ round starts, CONS(i) for all the active nodes is increased by 1 to keep track of the no of consecutive rounds node $n_{i}$ is active. After this update, the tree reconstruction phase will be over, and the nodes can resume their task to sense and send data packets to the sink. For each packet being sent to the sink by the node $n_{i}$, PT(i) is increased by 1 . This whole process is shown in algorithm 1 .

\section{Simulation Results}

In this paper, the performance of the proposed protocol is measured using the Castalia simulator. In this simulation, we have taken the number of nodes varies from 100 to 500. The nodes are deployed randomly using a uniform distribution approach. For each sensor node, the sensing radius energy 
Function: TIMER_2:

if $n(i)$ sleep constraint satisfied then

starts the process to sleep; (set timer of start_process) else

do nothing until next round

end if

End_of_Function: TIMER_2

Function: start_process:

ss(i) $=$ True

Send FIND_PARENT packet to notify N(j) (their child) that they need to

find new parent.

After a random time interval it triggers "determine_isActive".

End of Function:In start process

if N(j) receives FIND_PARENT packet then

if $\operatorname{ALIVE}(\mathrm{N}(\mathrm{j})) \operatorname{AND}[\mathrm{PFj}, 1==n(\mathrm{i}) \mathrm{OR} P F j, 2==n(i)]$ then

Broadcasts a WANT_PARENT packet.

Waits a random time while it chooses the best parent reply. end if

end if

if $\mathrm{N}(\mathrm{k})$ receives WANT_PARENT packet then

if $(\operatorname{ALIVE}(\mathrm{N}(\mathrm{k}))==$ true and $\mathrm{SS}(\mathrm{N}(\mathrm{k}))==$ false $)$ and $(\operatorname{LEVEL}(\mathrm{N}(\mathrm{k}))$

$==\operatorname{LEVEL}(\mathrm{N}(\mathrm{j}))$ or $\operatorname{LEVEL}(\mathrm{N}(\mathrm{k}))+1==\operatorname{LEVEL}(\mathrm{N}(\mathrm{j}))$ then broadcast PARENT_REPLY packet

end if

end if

if $\mathrm{N}(\mathrm{j})$ receives PARENT_REPLY packet then

if if $\mathrm{N}(\mathrm{j})$ wants new parent or $\mathrm{PFj}, 1 / \mathrm{PFj}, 2$ is -1$)$ then if $[\operatorname{LEVEL}(\mathrm{N}(\mathrm{k}+1))==\operatorname{LEVEL}(\mathrm{N}(\mathrm{l}))]$ OR $[\mathrm{if}(\mathrm{RR}(\mathrm{N}(\mathrm{k}))>\mathrm{RR}(\mathrm{N}(\mathrm{l}))$ AND $(\operatorname{LEVEL}(\mathrm{N}(\mathrm{k}))==\operatorname{LEVEL}(\mathrm{N}(\mathrm{l})))]$ then

Select node $\mathrm{N}(\mathrm{k})$ as parent over $\mathrm{N}(\mathrm{l})$

$$
\text { end if }
$$

$$
\text { end if }
$$

end if

if $\mathrm{N}(\mathrm{k})$ receives ACKNOWLEDGEMENT packet then becomes active

end if

Function: Determine_isActive:

finds no of child

if no of child $==0$ then

turns to sleep)

else

broadcasts MINUS ONE packet which instructs $\mathrm{N}(\mathrm{j}$ ) (its child) to use alternate parent field as primary parent field

waits a random time

finds no of child

if no of child $==0$ then

else

turns to sleep

remains active

end if

end if

if $\mathrm{N}(\mathrm{j})$ receives MINUS ONE packet then

if $\mathrm{PFj}, 1 !=\mathrm{PFj}, 2$ then

make the parent which is not $n(i)$ as primary parent field $\mathrm{PFj}, 1$. set $\mathrm{PFj}, 2$ as -1 . end if

end if

End_of_Function: Determine_isActive

Algorithm 1: The tree reconstruction phase between two consecutive rounds

consumption, transmission power, etc., are considered as per the detail given in TelosB data sheet [31].

Figure 6 shows the percentage of nodes active in each level of different size networks. The networks reach a maximum level of 3. Level-1, being the closest one to the sink, have the highest percentage of active nodes, while the number of active nodes falls considerably in lower levels. In level-3, there are almost negligible active nodes as most of the nodes present in this level are leaf nodes.

Figure 7 shows the number of active nodes in each level

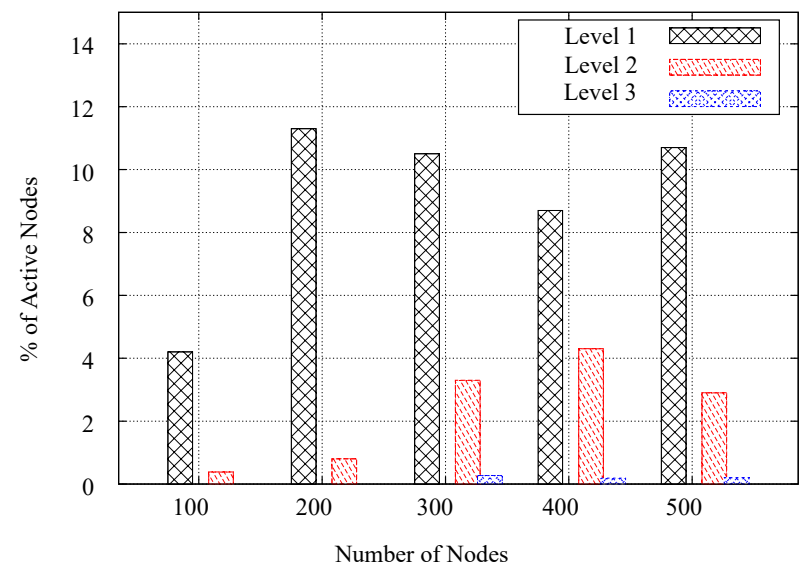

Fig. 6. Active Node in different level at different size network

of a network size of 500 deployed in an area of $150 \times 150 \mathrm{~m}^{2}$. This shows an average of 55 number of active nodes in level1 , around 15 number of active nodes in level-2. Initially, there are few numbers of active nodes in level-3, but as the tree construction progresses, the number of active nodes in level_3 falls to zero as most of them become leaf nodes. As the level wise tree construction procedure has been followed, there are crests and troughs in the graph pattern.

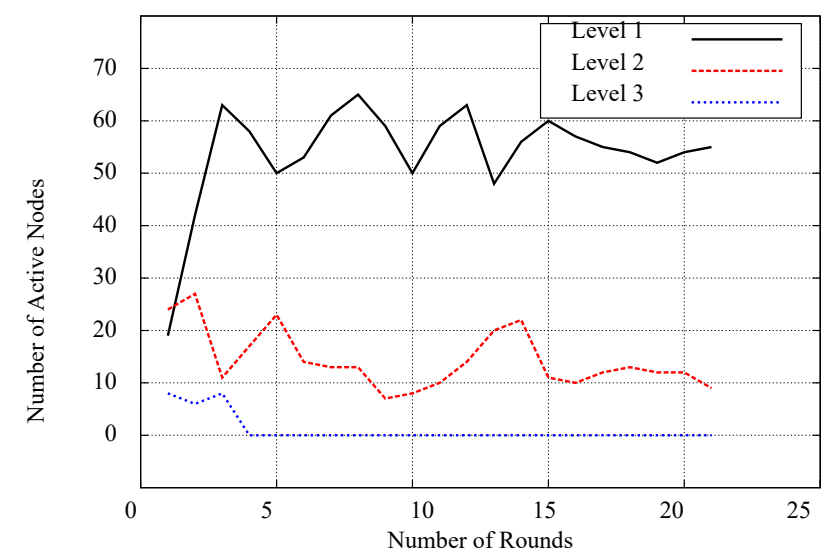

Fig. 7. Level wise active nodes in a WSN having 500 nodes

Figure 8 shows that in a 500 size network, the number of active nodes and the number of nodes out of the active nodes which have consumed significant energy during that round and meets the eligibility criteria to go to sleep in the next round. On an average of 70 active nodes, 30 to 40 nodes are opting to go to sleep in the next round.

Figure 9 shows the lifetime of the nodes in a WSN having 400 nodes deployed in a squared and rectangular area. As per this graph, in both the cases, the nodes start dying from the 26th round onward. The maximum level reached in the rectangular area is 5 as compared to 3 in the squared area. Similarly, Figure 10 shows that the proposed level wise approach increases the longevity of the network as compared 


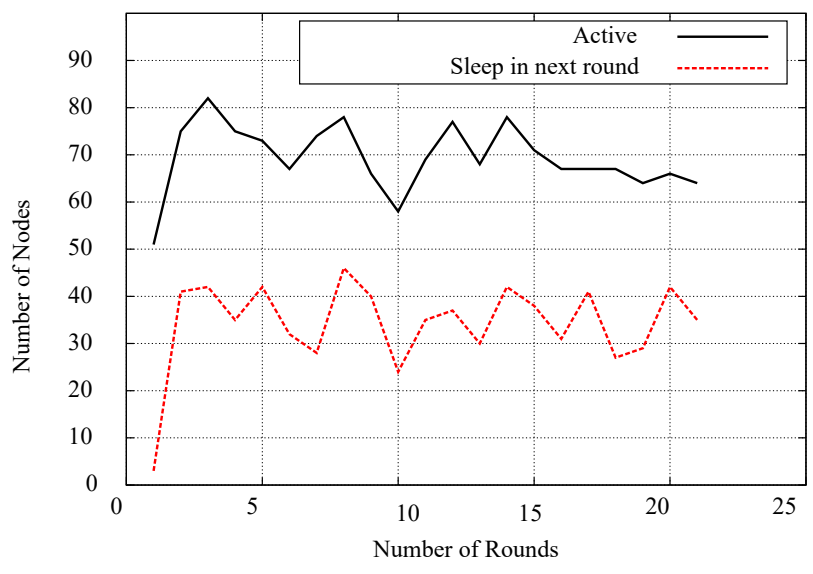

Fig. 8. Active node vs sleep node in next round in a WSN having 500 nodes

to earlier non level wise approach.

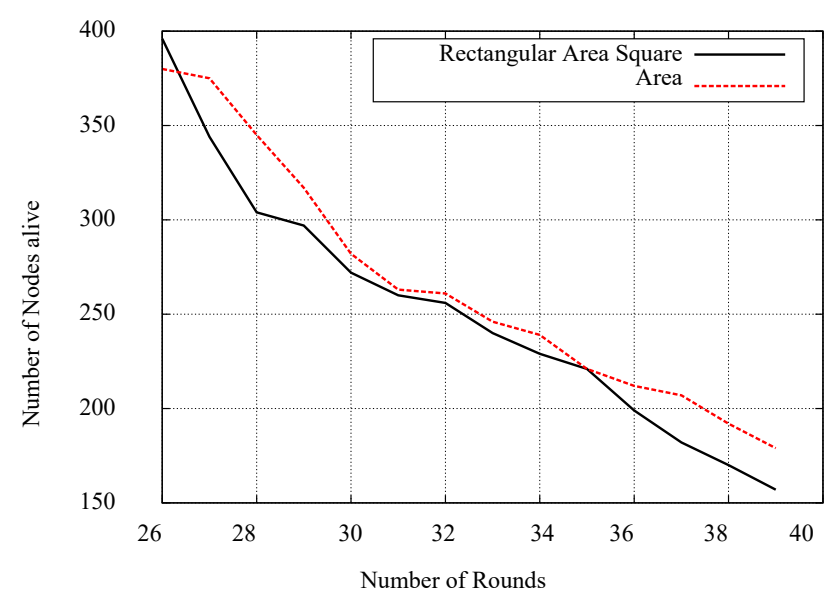

Fig. 9. Number of nodes Alive in a network size of 400 nodes deployed in square area and rectangular area

Figure 11 shows a comparison between a non level wise approach[29] and the proposed approach with respect to the number of nodes alive in different size networks at different rounds. It is seen that in every scenario, the number of nodes alive in level wise approach is more than that in the earlier non level wise approach.

Figure 12 shows the number of nodes alive in different size networks when the data packets are transmitted from the whole network vs. the data packets is transmitted from a particular region of WSN. From this figure, it is clear and obvious that the network lifetime will be more when the data packets are transmitted from a specific region of the network.

\section{CONCLUSiON}

In this paper, we addressed the requirement of minimum energy consumption in WSNs and proposed a level wise sleep scheduling mechanism along with connectivity that achieves the above requirement. In this proposed approach, a routing tree is built to maintain connectivity, and further, it is mended

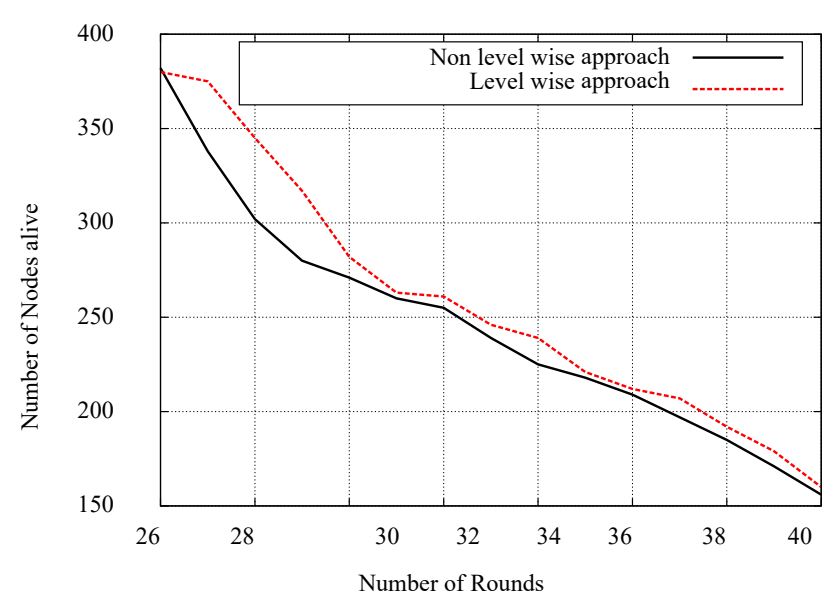

Fig. 10. Number of nodes Alive in a network size of 400 nodes deployed in non level wise approach vs. proposed approach.

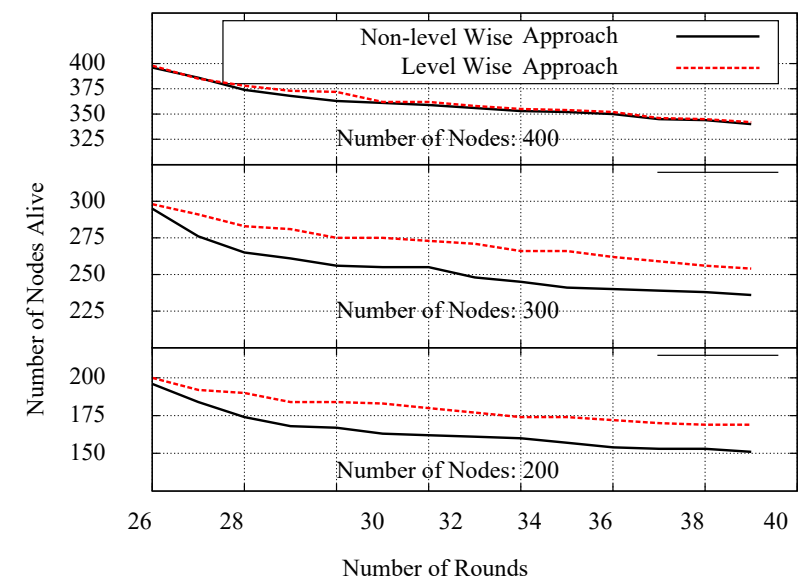

Fig. 11. Number of alive nodes in different size WSN in non level wise approach vs. the proposed approach

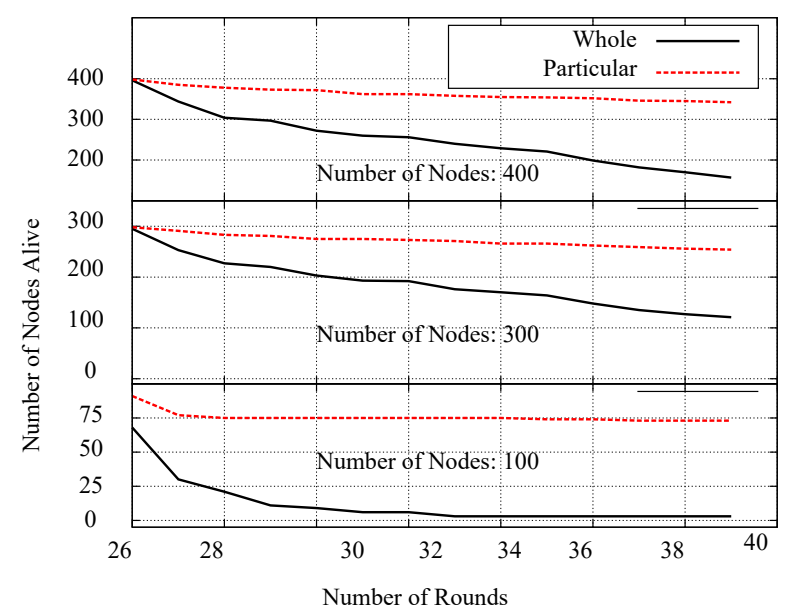

Fig. 12. Number of alive nodes in different size WSN where packets are transmitted from the whole network vs. specific region of the network 
periodically in parts to enhance the overall network lifetime along with ensuring equal power consumption among all the nodes in the network. Since the nodes are periodically changing their state in between active and sleep, in order to avoid the network hole, the internal node or the currently active node must communicate with its children before getting deactivated. The correct care has been taken to hand over the responsibility of its children to another active node so that a network hole will not be formed. The generated results proved that the overall energy consumption in the network is minimized and simultaneously maintain connectivity. Still, more comprehensive analysis of detecting network holes and its avoidance may be included as a future scope.

\section{REFERENCES}

[1] Vehbi Gungor, Gerhard P. Hancke: Industrial wireless sensor networks: Challenges, design principles, and technical approaches, Industrial Electronics, IEEE Transactions on, vol. 56, no. 10, pp. 4258-4265, 2009, doi: 10.1109/TIE.2009.2015754.

[2] Raghavendra Kulkarni, Anna Forster, and Ganesh Kumar Venayagamoorthy: Computational intelligence in wireless sensor networks: A survey, Communications Surveys Tutorials, IEEE, vol. 13, no. 1, pp. 68-96, 2011 , doi: 10.1109/SURV.2011.040310.00002.

[3] Sandra Compte S., Jaime Lloret, Miguel García Pineda, and José Francisco Toledo Alarcón: Power saving and energy optimization techniques for wireless sensor networks, Journal of Communications, vol.6, no.6, pp.439-459, 2011, doi: 10.4304/jcm.6.6.439-459.

[4] Ousmane Diallo, Joel JPC Rodrigues, Mbaye Sene, and Jaime Lloret: Distributed database management techniques for wireless sensor networks, IEEE Transactions on Parallel and Distributed Systems, vol. 6 , no. 2 pp. 604-620, 2013, doi: 10.1109/TPDS.2013.207.

[5] Olutayo Boyinbode, Hanh Le, and Makoto Takizawa: A survey on clustering algorithms for wireless sensor networks, International Journal of Space Based and Situated Computing, vol. 1, no. 2, pp. 130-136, 2011, doi: $10.1109 / \mathrm{NBiS} .2010 .59$.

[6] Nauman Aslam, William Phillips, William Robertson, and Shyamala Sivakumar: A multi criterion optimization technique for energy efficient cluster formation in wireless sensor networks, Information Fusion, vol. 12, no. 3, pp. 202-212, 2011, doi: 10.1016/j.inffus.2009.12.005.

[7] Wei Ye, John Heidemann and Deborah Estrin: An energy efficient MAC protocol for wireless sensor networks, in: Proceedings of IEEE Infocom, vol. 3, pp. 1567-1576, 2002, doi: 10.1109/INFCOM.2002.1019408.

[8] Guoliang Xing, Xiaorui Wang, Yuanfang Zhang, Chenyang Lu, Robert Bryan Pless, and Christopher Gill: Integrated coverage and connectivity configuration for energy conservation in sensor networks, ACM Trans. Netw., vol. 1, no. 1, pp. 36-72, Aug. 2005, doi: 10.1145/1077391.1077394.

[9] Honghai Zhang and Jennifer C. Hou: Maintaining sensing coverage and connectivity in large sensor networks, Ad Hoc Sensor Wireless Netw., vol. 1, nos. 1,pp. 89-124, 2005, doi: 10.1201/9780203323687.

[10] Hari Prabhat Gupta and Seela Veerabhadreswara Rao: Demand based coverage and connectivity preserving routing in wireless sensor networks, IEEE Syst. J., vol. 10, no. 4, pp. 1380-1389, Dec. 2016, doi: 10.1109/JSYST.2014.2333656.

[11] Muhammad Akhlaq, Tarek R. Sheltami, and Elhadi M. Shakshuki:C3: an energy efficient protocol for coverage, connectivity and communication in WSNs, Personal Ubiquitous Comput. vol-18, no-5, pp. 1117-1133, June, 2014,doi: 10.1007/s00779-013-0719-2.

[12] Jun-Bae Seo: Minimizing Energy Consumption of Duty Cycled Wireless Sensors, IEEE Communications Letters, vol. 19, no. 10, pp. 1698-1701, October 2015, doi: 10.1109/LCOMM.2015.2459698.

[13] P. Subramanian and E. Logashanmugam: Potentiality Timeserving Reliable Routing (PTRR) algorithm in multi hop wireless Sensor networks, published in Research Journal of Applied Sciences, Engineering and Technology, vol.9, no.3, pp. 172-175, January 2015, doi: 10.19026/rjaset.9.1392.

[14] P. Subramanian and E. Logashanmugam: Steadfast Routing for Increasing Reliability in Multi hop Wireless sensor networks, published in International Journal of Applied Engineering Research (IJAER), ISSN 0973-4562, vol. 9, no. 23, pp. 23345-23358, 2014.
[15] Junyoung Heo, Jiman Hong, Yookun Cho: EARQ: Energy aware routing for real time and reliable communication in wireless industrial sensor networks, Industrial Informatics, IEEE Transactions on, vol. 5, no. 1, 311, 2009, doi: 10.1109/TII.2008.2011052.

[16] Hongju Cheng, Zhihuang Su, Dafang Zhang, Jaime Lloret, Zhiyong Yu: Energy efficient node selection algorithms with correlation optimization in wireless sensor networks, International Journal of Distributed Sensor Networks, vol. 10, np. 3, 2014, doi: 10.1155/2014/576573.

[17] T.S. Rappaport: Wireless Communications: Principles and Practices, Prentice Hall, 1996, ISBN:978-0-13-042232-3.

[18] Robin Kravets and Parameshwaran Krishnan: Power Management Techniques for Mobile Communication, Proc. ACM MobiCom, pp. 157-168, 1998, doi: 10.1145/288235.288276.

[19] Volkan Rodoplu and Teresa H. Meng: Minimum Energy Mobile Wireless Networks, Proc. IEEE Int'l Conf. Comm. (ICC), vol. 5, no. 10, pp. 1432-1447 1998, doi: 10.1109/49.779917.

[20] Xiangyang Li, Wenzhan Song, and WeiZhao Wang: A Unified Energy Efficient Topology for Unicast and Broadcast, Proc. ACM MobiCom, pp. 1-15, 2005, doi: 10.1145/1080829.1080831.

[21] Peng-Jun Wan, Gruia Călinescu, Xiangyang Li, and Ophir Frieder: Minimum Energy Broadcast Routing in Static Ad Hoc Wireless Networks, ACM Wireless Networks, vol. 8, pp. 607-617, 2002, doi: 10.1109/INFCOM.2001.916310.

[22] Yu Wang, WeiZhao Wang, and Xiangyang Li: Distributed Low Cost Backbone Formation for Wireless Ad Hoc Networks, Proc. ACM MobiHoc, pp. 2-13, 2005, doi: 10.1145/1062689.1062692.

[23] XuFei Mao, Shaojie Tang, Xiaohua Xu, Xiang Yang Li, and Huadong Ma: Energy Efficient Opportunistic Routing in Wireless Sensor Networks, IEEE Trans. Parallel Distrib. Syst., vol. 22, no. 11, pp. 1934-1942, Nov. 2011, doi: 10.1109/TPDS.2011.70.

[24] Holger Karl and Andreas Willig: Protocols and architecture for WSN, John wiley and sons, 1td, 2006, ISBN: 978-0-470-09511-9.

[25] Amulya Ratna Swain, R. C. Hansdah, and Vinod Kumar Chouhan: An energy aware routing protocol with sleep scheduling for wireless sensor networks, In 24th IEEE International Conference on Advanced Information Networking and Applications, AINA 2010, Perth, Australia, vol. 20, no. 13, pp. 933-940, 2010, doi: 10.1109/AINA.2010.11.

[26] Udit Narayan Kar, Dipti Dash, Debarshi Sanyal, Dibyajyoti Guha, and Samiran Chattopadhyay: A Survey of Topology Transparent Scheduling Schemes in Multi Hop Packet Radio Networks, IEEE Communications Surveys \& Tutorials, vol. 19, no. 4, PP. 2026-2049, August, 2017, doi: 10.1109/comst.2017.2739418.

[27] Subhasis Dash, Saras Kumar, Manas Lenka, and Amulya Ratna Swain: Multi Token Based MAC-Cum-Routing Protocol for WSN: A Distributed Approach, Journal of Communications Software and Systems, Journal of Communications Software and Systems, Vol-15, no. 3, doi10.24138/jcomss.v15i3.709.

[28] Nachiketa Tarasia, Manas Ranjan Lenka, and Amulya Ratna Swain : Energy Efficient Extended Coverage in Wireless Sensor Networks, 2015 IEEE 29th International Conference on Advanced Information Networking and Applications, Gwangiu, pp. 247-254, 2015, doi: 10.1109/AINA.2015.192.

[29] Subhasis Dash, Satya Mallick, R. Hansdah, and Amulya Ratna Swain : A Distributed Approach to Construct Hierarchical Structure for Routing with Balanced Energy Consumption in WSNs, 2015 IEEE 29th International Conference on Advanced Information Networking and Applications, Gwangiu, pp. 382-388, 2015, doi: 10.1109/AINA.2015.210.

[30] G. Pradeebaa and N. Lavanis: Network lifetime improvement using routing algorithm with sleep mode in wireless sensor network, 2016 International Conference on Wireless Communications, Signal Processing and Networking (WiSPNET), Chennai, pp. 1572-1575, 2016 doi: 10.1109/WiSPNET.2016.7566401.

[31] Telosb data sheet. http://www.xbow.com/Products/ Product pdf files/Wireless pdf/TelosB Datasheet.pdf

[32] Ahmad Aiman Shallahuddin, Mohd Fadzil Abdul Kadir, Mohamad Afendee Mohamed, Ahmad Faisal Amri Abidin, Nor Surayati Mohamad Usop, and Zahrahtul Amani Zakaria: An Enhanced Adaptive Duty Cycle Scheme for Optimum Data Transmission in Wireless Sensor Network, Information Science and Applications. Springer, Singapore, pp. 33-40, 2020, doi : 10.1007/978-981-15-1465-4.4

[33] Xianlong Jiao, Wei Lou, Songtao Guo, Libin Yang, Xinxi Feng, Xinxi Feng, and Guirong Chen: Delay Efficient Scheduling Algorithms for Data Aggregation in Multi Channel Asynchronous Duty Cycled WSNs, IEEE Transactions on Communications vol. 67, no.9, pp. 6179-6192, 2019, doi: 10.1109/TCOMM.2019.2924222.

[34] Runze Wan, Naixue Xiong: An energy efficient sleep scheduling mechanism with similarity measure for wireless sensor networks Human 
centric Computing and Information Sciences, vol. 8 , no. 18, 2018, doi: 10.1186/s13673-018-0141-x.

[35] Miguel Lino, Vitor Vasconcelos, Álvaro Ian, Erico Leão, André Soares, and Carlos Montez: An Efficient Mechanism to Improve Convergecast Traffic in Cluster tree Wireless Sensor Networks Based on IEEE 802.15. 4 IECON 2019-45th Annual Conference of the IEEE Industrial Electronics Society. Vol. 1, pp. 2811-2816, 2019, doi: 10.1109/IECON.2019.8927736.

[36] Louie Chan, Karina Gomez Chavez, Heiko Rudolph, and Akram Hourani: Hierarchical routing protocols for wireless sensor network: a compressive survey., Wireless Networks, pp. 1-24, 2019, doi: $10.1007 / \mathrm{s} 11276-020-02260-\mathrm{z}$

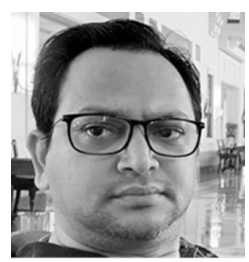

Nachiketa Tarasia received his M.Tech degree in Computer Science and Engineering from CET, BPUT, Bhubaneswar, India in 2007. Currently, he is working as an Assistant Professor in the School of Computer Engineering, KIIT Deemed To Be University, Bhubaneswar, India.His research interests include wireless sensor networks, Computer Network and Algorithm Design.

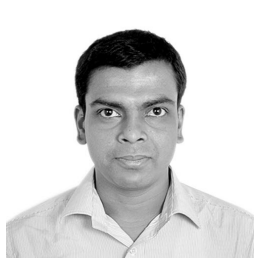

Amulya Ratna Swain received his M.E. Degree in Software Engineering from Jadavpur University, Calcutta, India in 2006. He received the Ph.D. degree in Computer Science from Indian Institute of Science, Bangalore, India, in 2013. Currently, he is working as an associate professor in the School of Computer Engineering, KIIT Deemed To Be University, Bhubaneswar, India. His research interests include wireless sensor networks, distributed computing, and operating systems.

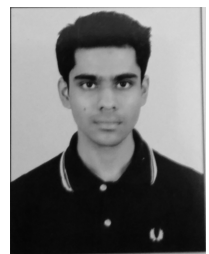

Soham Roy received his B.Tech in Computer Science and Engineering from School of Computer Engineering, Kalinga Institute of Industrial Technology (KIIT) University.His primary research interest was on sensor nodes, Machine Learning and financial modelling.

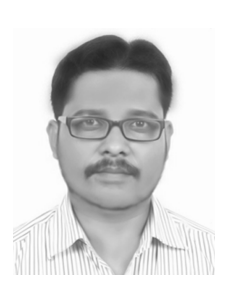

Udit Narayana Kar received the Bachelor of Technology degree in information technology and engineering from Biju Patnaik University, Rourkela, India, in 2008 and the Master of Technology degree in computer science and engineering from the Institute of Technical Education and Research, SOA University, Bhubaneswar, India, in 2012. He is currently a Research Scholar with the School of Computer Engineering, KIIT University, Bhubaneswar. He has four years of experience in education and research. He was a Research Fellow in a UGC sponsored project from 2012 to 2014. His major areas of research interest include $5 \mathrm{G}$ wireless communications, scheduling in wireless networks, and device to device communication. He is a Life Member of the Institution of Electronics and Telecommunication Engineers. 